\title{
Titles of Papers Presented at the 40th Annual Meeting of the Herpetological Society of Japan (10-11 November 2001 at Niigata University, Niigata)
}

Abstracts in Japanese will appear in Bulletin of the Herpetological Society of Japan, Vol. 2002, No 1.

[Turtles]

1. Ecology of Trachemys scripta on Okinawa Island. By: Dai Hatanaka, Takeshi Sasaki, Yoshitsugu Kawashima, and Go Ogura.

2. The relationship between size distribution and growth rate of hawksbill turtles, Eretmochelys imbricata, in the water around Vadoo Island, Maldives. By: Naoki Kamezaki, Izumi Sakamoto, and Tokihiko Sakamoto.

3. Distribution and population structure of the common snapping turtle, Chelydra serpentina, in Inbanuma Basin. By: Raita Kobayashi, Noriyuki Hashimoto, and Masami Hasegawa.

4. In which seasons do Chinemys reevesii and Mauremys japonica grow? By: Yasuhiro Kosuge and Masami Hasegawa.

5. The factor which causes weight difference in the Japanese pond turtle, Mauremys japonica, among different ponds. By: Hideki Noda and Naoto Kamata.

6. Intersexual dietary differences in a feral population of the Chinese soft-shelled turtle, Pelodiscus sinensis. By: Hiroyuki Sato, Atsushi Kaneko, and Hidetoshi Ota.

7. Female-biased mortality and it's consequence on adult sex ratio in the Chinemys reevesii on an island. By: Toshiaki Takenaka and Masami Hasegawa.

8. Comparison of the shell formation pattern between Caretta caretta and Pelodiscus sinensis. By: Masayoshi Tokita and Shigeru Kuratani.

9. Phylogenetic relationships and biogeography of testudinid turtles. By: Yuichirou
Yasukawa, Hisashi Kimoto, and Akira Taketo.

[Lizards]

10. Molecular evolution of $\rho B$-crystallin of the gecko, Lepidodactylus lugubris. By: Yutaka Fujii, Hisashi Kimoto, Keiko Ishikawa, Yuichirou Yasukawa, Saiko Yamashiro, Kikuko Watanabe, and Akira Taketo.

11. Ecological observations of the chameleons Frucifer oustaleti and $F$. rhinoceratus in a dry forest of western Madagascar. By: Masami Hasegawa, Akira Mori, and Isami Ikeuchi.

12. Phylogenetic relationships of water skinks of the genus Tropidophorus (Reptilia: Scincidae). By: Tsutomu Hikida, Masanao Honda, and Hidetoshi Ota.

13. Molecular evaluation of population systematics and biogeography of Ateuchosaurus pellopleurus. By: Masanao Honda.

14. Natural history of the day gecko, Phelsuma madagascariensis kochi, in the dry forest of Madagascar. By: Isami Ikeuchi, Akira Mori, and Masami Hasegawa.

15. Geographic variation in behavioral responses of the lizard (Eumeces okadae) to the odor from a snake-predator. By: Hideo Nakazawa, Akira Mori, and Masami Hasegawa.

16. The distribution of Eumeces latiscutatus and E. okadae (Reptilia: Scincidae) on the Japanese main islands. By: Taku Okamoto, Tsutomu Hikida, and Junko Motokawa.

17. Taxonomic status of a gecko of the genus Gekko from Belau (Squamata: Gekkonidae). By: Hidetoshi Ota.

18. An undescribed species of the genus Pseudoacontias (Reptilia: Scincidae) from 
Nosy Be Island, Madagascar. By: Shuichi Sakata and Tsutomu Hikida.

19. Reproduction of Takydromus smaragdinus on Uke Island, the Amami-shoto. By: Sen Takenaka, Hajime Moriguchi, and Toshihiko Hayashi.

[Snakes]

20. Behavioral responses to the introduced toxic prey, Bufo marinus, by the native snakes of the Ryukyus. By: Noriko Kidera and Hidetoshi Ota.

21. Periodical foraging migration of Ovophis okinavensis: individual movements. By: Akira Mori, Mamoru Toda, and Hidetoshi Ota.

22. Accidents caused by foreign snakes in this year. By: Michihisa Toriba.

[Salamanders]

23. Food habits of Andrias japonicus in small streams, Tottori Prefecture. By: Sumio Okada.

24. Utilization of alternative breeding sites of the Hokkaido salamander, Hynobius retardatus. By: Takanori Sato and Kimihiro Tsutsumi.

25. Tracing terrestrial movement of Hynobius lichenatus with radio-telemetry II. By: Hiroshi Ota.

26. Taxonomic status of Hynobius tenuis. By: Kanto Nishikawa and Masafumi Matsui.

27. External changes of larval salamanders (Hynobius tokyoensis) as induced by differential water flow conditions. By: Sadao Ihara.

28. Chromosomal polymorphism of the nucleolus organizer regions in Hynobius nigrescens. By: Chikako Ikebe and Sei-ichi Kohno.

29. Variation of Hynobius naevius in northern Kyushu, Japan. By: Atsushi Tominaga, Masafumi Matsui, Kanto Nishikawa, and Shin'ichi Sato.

30. Breeding sites of Hynobius kimurae in Hachioji, Tokyo. By: Yasuchika Misawa and Masafumi Matsui.
31. Seasonal changes of sperm storage within the spermatheca of the newt Cynops pyrrhogaster. By: Shigeharu Akiyama.

32. Heredity of color phenotypes of Cynops pyrrhogaster. By: Kumi Matsui, Junsuke Marunouchi, Yoshinori Hasegawa, and Masahisa Nakamura.

33. What types of males are chosen by females in the Japanese newt, Cynops pyrrhogaster ? By: Takako Kutsuki and Eisuke Hasegawa.

34. Anatomical observation of the tongue and associated muscles in the newt, Cynops pyrrhogaster. By: Yoshihito Yamazaki.

[Frogs and toads]

35. Migrations of Bufo torrenticola and $B$. japonicus formosus during the breeding season around Nozumi River, Toyama Prefecture. By: Hisao Nambu, Tamotsu Fukuda, and Yoshimasa Araki.

36. Observations on the cane toad Bufo marinus on Iriomote Island. By: Mitsuhiko Toda and Masaya Tatara.

37. The polymorphic nucleolar organizer region and its mode of inheritance in Hyla japonica. By: Kunio Sekiya, Takashi Fujii, and Kenji Moriwaki.

38. The present condition of about 200 breeding sites of Rana japonica and $R$. ornativentris reported from Tochigi, Gunma, and Saitama Prefectures in the 1970's. By: Terutake Hayashi, Hajime Moriguchi, Katsuhiro Tomioka, and Yuki Kimura.

39. Physical contact with larval salamanders (Hynobius retardatus) induces morphological changes in frog tadpoles (Rana pirica). By: Osamu Kishida and Kinya Nishimura.

40. Geological environment of breeding sites of Rana tagoi. By: Jun'ichi Ohki.

41. On two types of Rana tagoi tagoi from Kitayama, Kyoto. By: Tadafumi Sugihara, Kanto Nishikawa, Takahiro Sugahara, and Masafumi Matsui.

42. Life of Rana tagoi of the Boso Peninsula, Chiba Prefecture. By: 
Takehito Ueda, Jun'ichi Ohki, Masami Hasegawa, and Tamotsu Kusano.

43. The theoretical model of seasonal sex ratio in Rana rugosa. By: Yukio Sakisaka, Hiroyasu Amagai, and Hitoshi Yoshimura.

44. The occurrence of Rana rugosa with mid-dorsal stripe in the Atsumi Peninsula. By: Takashi Yabe, Tomoya Asaka, and Tamotsu Kusano.

45. Microscopic anatomy of the frog, Rana nigromaculata, with special reference to the skin and some digestive organs. By: Masahiko Kumakura, Norio Yoshie, and Hiroshi Kobayashi.

46. Seasonal changes in number of Rana porosa brevipoda killed on the road. By: Takuo Sawahata.

47. Genetic variation in Rana nigrovittata within Thailand. By: Masafumi Matsui, Kanto Nishikawa, and Wichase Khonsue.

48. Factors affecting male mating success in Rana kuhlii in Taiwan. By: Hiroshi Tsuji.

49. A preliminary study of genetic differentiation in the Japanese tree frog, Rhacophorus arboreus. By: Seiji Ikeda, Masafumi Matsui, and Tomoko Tanaka-Ueno.

50. Geographical distribution of the nucleolar organizer region polymorphism in Rhacophorus schlegelii. By: Tadashi Togashi and Kunio Sekiya.
51. Comparisons of digestive tract morphology between the oophagous tadpole of Chirixalus eiffingeri and tadpoles of other rhacophorid species. By: Yoko Gima, Wen-hao Chou, and Hidetoshi Ota.

52. Factors affecting the movements of anurans in fallow paddy fields. By: Kinji Fukuyama.

53. Motility of alimentary tract and its ontogenetic variation in tadpoles. By: Masamichi Yamashita, Akiko Yamashita, Tomio Naitoh, Kerri Oseen, and Richard J. Wassersug.

[Amphibians general]

54. Prediction of amphibian community habitats in a large area. By: Satoshi Osawa, Takehiko Katsuno, Yoshiyuki Hioki, and Kenichi Matsubayashi.

55. Noninvasive sampling methods for genotyping in amphibians. By: Tomoko Tanaka-Ueno, Seiji Ikeda, and Masafumi Matsui.

[General]

56. Amphibians and reptiles in the Red Data Book of Nagasaki Prefecture, Japan. By: Takanori Matsuo. 